\title{
Die Langzeit-Sauerstoff-Therapie (LTOT) - Was sollten Arzt, Versorger und Krankenkasse wissen?
}

\author{
Long-Term Oxygen Therapy (LTOT) - What Should Physicians, Homecare- \\ Provider and Health Insurance Companies Know?
}

Autoren

Institute

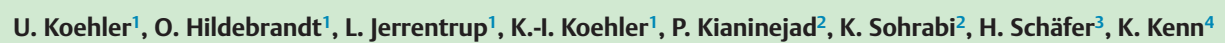

Die Institutsangaben sind am Ende des Beitrags gelistet. eingereicht 30.11 .2013 akzeptiert nach Revision 6.12 .2013

\section{Bibliografie}

Dol http://dx.doi.org/ 10.1055/s-0033-1359198 Pneumologie 2014; 68: 193-198

(c) Georg Thieme Verlag KG Stuttgart · New York ISSN 0934-8387

\section{Korrespondenzadresse Prof. Dr. Ulrich Koehler Klinik für Innere Medizin SP Pneumologie, Intensiv- und Schlafmedizin Universitätsklinikum Gießen und Marburg GmbH Standort Marburg Baldingerstraße 1 35043 Marburg koehleru@med.uni-marburg.de}

\section{Zusammenfassung $\nabla$}

Die Langzeit-Sauerstoff-Therapie (LTOT) führt bei Patienten mit chronisch respiratorischer Insuffizienz nachweislich zur Prognoseverbesserung. Pathogenetisch sind eine Verbesserung der Oxygenierung, eine Abnahme des pulmonal-arteriellen Drucks sowie eine Abnahme der Atemarbeit von Bedeutung. Aufgrund der Vielfalt und großen Unterschiedlichkeit der LTOT-Systeme ist eine individuelle Einstellung des Patienten auf die Langzeit-Sauerstoff-Therapie notwendig. Zu berücksichtigen sind dabei insbesondere Mobilitätsaspekte. Bei den Applikationsgeräten ist zu unterscheiden zwischen stationären/mobilen Flüssigsauerstoffsystemen sowie stationären/mobilen Konzentratoren. Die Sauerstofftitration sollte individuell, bezogen auf eine Ruhe- und eine Belastungsphase (z.B. 6-Minuten-Gehtest) sowie den Schlaf, vorgenommen werden. Der Einsatz von Spar- bzw. Demandventilen ist kritisch zu prüfen. Er ist nur aufgrund ärztlicher Verordnung gerechtfertigt und bedarf engmaschiger Kontrollen.

\section{Pathophysiologie der chronisch respiratorischen Insuffizienz \\ $\nabla$}

Das respiratorische Kontrollsystem dient zur Aufrechterhaltung der Säure-/Basenhomöostase, der Blutgase sowie der Steuerung der rhythmischen Kontraktion und Tonisierung der an der Atmung beteiligten Muskulatur. Dies muss in Anpassung an die spezifischen Leistungsanforderungen des Organismus geschehen. Die Kenngröße einer respiratorischen Insuffizienz ist das Verhalten der arteriellen Blutgase, wobei die Hypoxämie als respiratorische Partialinsuffizienz Ausdruck einer Oxygenierungsstörung und die Hyperkapnie als respiratorische Globalinsuffizienz Ausdruck einer ventilatorischen Störung sind. Bronchopulmonale Erkrankungen können langfristig zu einer Störung von Gasaustausch und Atemmechanik und

\section{Abstract \\ $\nabla$}

Long-term oxygen treatment (LTOT) has been demonstrated to improve prognosis in patients with chronic respiratory insufficiency. In terms of pathogenesis, improved oxygenation, reduction of pulmonary artery pressure as well as reduction of respiratory work are important. Since there are considerable differences between the LTOT systems, individually tailored therapy is needed. In particular, the mobility aspects of the patients must be taken into consideration. It is important to distinguish between stationary/mobile devices with a liquid oxygen system and stationary/mobile devices with oxygen concentrator. Oxygen titration should be performed in relation to rest and activity phases (e.g. 6 minute walk test) as well as in relation to the sleep phase. Employing devices with demand-controlled valves should be critically examined. This can be undertaken only under physician orders and requires continuous monitoring.

damit zu einer chronisch respiratorischen Insuffizienz führen [1]. Pathogenetisch kann eine Hypoxämie durch eine Reduzierung der Gasaustauschfläche, eine Störung der alveolo-kapillären Diffusion sowie eine Perfusionsstörung oder eine Kombination dieser Mechanismen bedingt sein. Die respiratorische Partialinsuffizienz stellt meist den Beginn einer unzureichenden Sauerstoffversorgung des Organismus dar. Dabei können vor allem bei Patienten mit fibrosierenden Lungenerkrankungen Normalwerte in Ruhe einen gravierenden Sauerstoffmangel bei körperlicher Belastung maskieren. $\mathrm{Zu}$ beachten ist, dass ein Sauerstoffmangel, insbesondere wenn er sich langsam entwickelt, für den Betroffenen subjektiv oft kaum oder nicht spürbar ist. Somit kommt der exakten Anamnese mit Blick auf reduzierte Belastungsintensitäten und eingeschränkten Mobili- 
tätsradius große Bedeutung zu. Durch schrittweise Anpassung der Alltagsaktivitäten an die eigenen reduzierten Kapazitäten kann eine solche Entwicklung bis hin zu bedenklichen, nicht realisierten Limitationen der Oxygenierung führen.

Bei einer respiratorischen Globalinsuffizienz, der Kombination von Hypoxämie und Hyperkapnie, sind Faktoren wie das Vorliegen einer Funktionsstörung der Atemmuskulatur, Muskeldystrophien, thorakorestriktive Erkrankungen mit Beeinflussung der Atemmechanik, eine Reduktion des Atemantriebs sowie primäre oder sekundäre Hypoventilationssyndrome ursächlich von Bedeutung. Das Verhältnis von Belastung und Leistungsfähigkeit des respiratorischen Systems ist bei diesen Erkrankungen zu Ungunsten einer erhöhten Last verschoben. Die Überbeanspruchung bzw. Ermüdung der Atemmuskulatur bei hyperkapnischer respiratorischer Insuffizienz ist pathophysiologisch bedeutsam [1]. Eine vermehrte und dauerhafte Beanspruchung der Atemmuskulatur kann zu einer zunehmenden Erschöpfung der Muskelkraft bei erhöhtem Energieverbrauch führen. Anpassungsbedingt stellt der Organismus eine Schonatmung ein, die mit einer Erhöhung des $\mathrm{pCO}_{2}$ einhergeht.

Bei einem Teil der Patienten mit einer chronisch respiratorischen Insuffizienz kommt es erstmals während des Schlafes zur Manifestation von Hypoxämie und Hyperkapnie [2,3]. Schreitet die Erkrankung voran, sind die Blutgasalterationen in Form einer Globalinsuffizienz auch am Tage zu objektivieren. Patienten mit respiratorischer Globalinsuffizienz am Tage weisen insbesondere im REM-Schlaf Hypoxie- und Hyperkapniephasen auf [3]. Vor allem bei Patienten mit schwergradiger COPD und Lungenemphysem kommt es im REM-Schlaf zum Auftreten von langstreckigen Hypoventilationsphasen und ausgeprägten Blutgasveränderungen. Pathophysiologisch erklärt sich die Hypoventilation durch eine Zunahme der Atemarbeit bei REM-assoziiertem Ausfall der inspiratorischen Atemhilfsmuskulatur und veränderter Chemorezeptorsensitivität. Die respiratorische Globalinsuffizienz ist in der Regel den weit fortgeschrittenen Krankheitsstadien vorbehalten. Hier gilt es, den Nutzen einer LTOT subtil zu evaluieren bzw. in seltenen Fällen eine mögliche Gefährdung durch unkritische Sauerstoffgaben auszuschließen.

Die therapeutischen Optionen der chronisch respiratorischen Insuffizienz müssen notwendigerweise den pathophysiologischen Mechanismen Rechnung tragen. Dies bedeutet, dass neben der medikamentösen Therapie Maßnahmen zur Anwendung kommen müssen, die zur „Entlastung“ und „Erholung“ der Atemmuskulatur, zur Korrektur der Hypoxämie und zur Vermeidung schlafassoziierter Hypoventilationen beitragen.

In den letzten Jahren hat sich die Behandlungsstrategie der chronischen Lungenerkrankungen - insbesondere der COPD - dahingehend verändert, dass neben dem medikamentösen Ansatz vor allem der Bewegungstherapie eine grundlegende Bedeutung zugemessen wird $[4,5]$. Ambulante und stationäre Rehabilitation und Lungensport sind mittlerweile etablierte Therapiekonzepte für Patienten mit mittelgradiger und auch schwerer COPD. Das Risiko der Hospitalisierung sowie der Mortalität ist bei körperlich aktiven Patienten mit COPD signifikant geringer. Die Effekte der Sport- und Bewegungstherapie können jedoch, insbesondere bei Patienten mit schwergradigerer Erkrankung, nur dann aufrechterhalten werden, wenn ein regelmäßiges und der Erkrankung angepasstes körperliches Trainingsprogramm beibehalten wird. Das bedeutet zwangsläufig, dass bei den Patienten auch eine ausreichende Oxygenierung unter Belastung gewährleistet sein muss, die die Mobilität erhöht.
Die Trainingstherapie sollte dauerhaft zu einer gesteigerten körperlichen Aktivität im Alltag führen, was zumeist eine grundlegende Bewusstseinsänderung beim Patienten voraussetzt. Um häuslich aktiv und mobil zu sein und auch zu bleiben, ist eine mobile, den Alltagsbedingungen angepasste Sauerstoffversorgung notwendig.

\section{Die Langzeit-Sauerstoff-Therapie (LTOT) $\nabla$}

Basis der nichtmedikamentösen Therapie bei chronisch respiratorischer Insuffizienz mit relevanter Hypoxämie $\left(\mathrm{PaO}_{2}\right.$ in Ruhe $\leq 55 \mathrm{mmHg}$ ) stellt die Langzeitapplikation von Sauerstoff dar. Die Korrektur der Hypoxämie durch Erhöhung der inspiratorischen Sauerstoffkonzentration dient der Sicherstellung der zellulären Organfunktion. Dabei müssen jedoch weitere Faktoren wie eine ausreichende Hämoglobinkonzentration und eine ausreichende kardiale Pumpleistung berücksichtigt werden. Ziel der Sauerstofftherapie sollte es sein, einen $\mathrm{PaO}_{2}>60 \mathrm{mmHg}(7,98 \mathrm{kPa})$ oder eine Sauerstoffsättigung von $>90 \%$ sicherzustellen. Eine verbesserte Überlebensrate von Patienten mit COPD durch eine kontinuierliche Sauerstoffgabe wurde sowohl in der Medical Research Council, MRC-Studie [6] als auch in der Nocturnal Oxygen Therapy Trial, NOTT-Studie [7] nachgewiesen. Hinsichtlich der zugrundeliegenden Mechanismen wurde eine Verbesserung der pulmonalen Hämodynamik mit einer Verhinderung eines weiteren Anstiegs des pulmonalarteriellen Drucks (PAP) bzw. einer Abnahme des PAP beschrieben [8,9].

Studien zur Beurteilung des Effektes einer Langzeit-SauerstoffTherapie haben zeigen können, dass die Verlängerung der Lebenserwartung von Patienten mit chronisch respiratorischer Insuffizienz eindeutig positiv mit der Hyperkapnie und nicht mit der Ausprägung der Hypoxämie zu Beginn der Therapie bzw. deren Korrektur korreliert. Als diesen Ergebnissen zugrundeliegende pathophysiologische Rationale ist anzunehmen, dass die Sauerstoffapplikation zu einer Reduzierung des Atemminutenvolumens, sprich zu einer Hypoventilation mit geringer, konsekutiver Hyperkapnie, führt. Die Hypoventilation wird somit als therapeutischer Benefit interpretiert, zumal sie zu einer Entlastung der Atemmuskulatur und zu einer Regenerierung der Energiespeicher führt. Dieser Mechanismus kann auch als permissive Hyperkapnie bezeichnet werden.

In einer Studie bei COPD-Patienten mit schwerer Hypoxämie $\left(\mathrm{PaO}_{2}<55 \mathrm{mmHg}\right)$, bei denen eine Langzeit-Sauerstoff-Therapie eingeleitet wurde, zeigte sich eine schlechtere Prognose bei denjenigen Patienten, die eher dem „emphysematösen“ Typus entsprachen [10]. Eine verbesserte Prognose hingegen fand sich bei Patienten mit „bronchitischem“ Typus und mäßiger Hyperkapnie. Vergleichbare Ergebnisse mit inverser Relation zwischen Mortalität und $\mathrm{PaCO}_{2}$ bei Patienten mit chronisch obstruktiver Bronchitis ergab die Analyse des französischen ANTADIR-Registers [11]. In den Jahren 2014 und 2015 ist mit den Ergebnissen zweier großer Multizenterstudien zu rechnen, die sich mit dem Einfluss der LTOT auf die Prognose von Patienten mit COPD sowie Hypoxämie in Ruhe oder Belastung auseinandersetzen. Wahrscheinlich haben vor allem diejenigen Patienten einen prognostischen Zugewinn, bei denen die Langzeit-Sauerstoff-Therapie eine mäßige Hypoventilation mit Abnahme der Atemarbeit und Entlastung der Atemmuskulatur induziert. Es spricht einiges dafür, dass bestimmte Subgruppen der Patienten mit COPD von einer Langzeit-Sauerstoff-Therapie in besonderem Maße profitieren. Positive Effekte der Sauerstoffapplikation umfassen zudem eine 
Verbesserung der körperlichen Belastbarkeit und des neuropsychologischen Status. Eine leichtgradige respiratorische Globalinsuffizienz bzw. ein moderater Anstieg des $\mathrm{PaCO}_{2}$ unter Sauerstoffapplikation stellen, entgegen der früheren Lehrmeinung, sicher keine Kontraindikation für eine Langzeit-Sauerstoff-Therapie dar. Eine LTOT ist auch dann durchführbar, wenn die Patienten unter Sauerstoffgabe eine stabile chronische hyperkapnische Insuffizienz aufweisen. Bei einer ausgeprägteren $\mathrm{CO}_{2}$-Retention sollte zur Entlastung der Atemmuskulatur eine intermittierende Selbstbeatmungstherapie in Erwägung gezogen werden.

\section{Entscheidungskriterien zur LTOT}

Sauerstoff ist nach deutschem Gesetz ein Medikament und als solches nur vom Arzt zu verordnen. Anpassungen der $\mathrm{O}_{2}$-Therapie dürfen somit ebenfalls nur durch einen Arzt erfolgen, zumal unkritische Applikationen von Sauerstoff den Patienten akut gefährden können.

Die Kriterien der verschiedenen nationalen und internationalen Fachgesellschaften zur Einleitung der Langzeit-Sauerstoff-Therapie sind weitgehend einheitlich $[12,13]$. Wenngleich die Prognoseverbesserung durch die LTOT hauptsächlich bei Patienten mit COPD und chronischer Hypoxämie nachgewiesen wurde, so besteht Konsens darüber, dass diese Therapie bei allen Patienten mit Hypoxämie unabhängig von deren Genese durchgeführt werden sollte. Wesentlich ist, dass die Indikationsstellung in einer stabilen Phase der Grunderkrankung unter optimaler medikamentöser Therapie erfolgt. Erst wenn der Patient vier Wochen lang ausreichend medikamentös therapiert worden ist und keine Verbesserung der Blutgaswerte eintritt, sollte eine LTOT eingeleitet werden. Die Blutgase sollten mittels arterieller oder kapillärer Blutgasanalyse dokumentiert und die erforderlichen $\mathrm{O}_{2}$-Flussraten durch eine entsprechende Testung ermittelt werden. Darüber hinaus sollte bei mobilen Patienten eine Überprüfung der Blutgase unter körperlicher Belastung erfolgen. Falls möglich sollte auch die notwendige $\mathrm{O}_{2}$-Flussrate für die Nacht eruiert werden. Ziel ist die Sicherstellung eines stabilen $\mathrm{PaO}_{2}>60 \mathrm{mmHg}$. Die minimale Sauerstoffapplikationsdauer sollte unter Einschluss der Schlafzeit 16 Stunden nicht unterschreiten, da ein prognostischer Effekt von der täglichen Therapiedauer abhängt. Dabei ist allerdings zu beachten, dass innerhalb dieses anzustrebenden Zeitraums die Phasen des größten Sauerstoffbedarfs, nämlich der körperlichen Aktivität, eingeschlossen sind. Dies wird im klinischen Alltag oft missverstanden, indem die Nachtruhe und Zeiten von Inaktivität zu 16 Stunden aufaddiert werden.

Für die ausschließliche Therapie nächtlicher Hypoxämien mit Sauerstoff bei Patienten mit einem $\mathrm{PaO}_{2} \geq 55 \mathrm{mmHg}$ am Tage ohne Zeichen der Rechtsherzbelastung bzw. Polyglobulie gibt es hinsichtlich der Mortalitätsabnahme zwar keine Evidenz, dennoch wird diese in den deutschen Leitlinien [12] empfohlen, wobei auf eine Senkung des mittleren pulmonal-arteriellen Drucks um 3,7 mmHg hingewiesen wird. Eine nächtliche Sauerstofftherapie hatte bei 76 COPD-Patienten mit mäßiger Hypoxämie am Tage $\left(\mathrm{PaO}_{2}\right.$ 56-69 mmHg) und nächtlichen Desaturationen im randomisierten Vergleich über einen Zeitraum von 3 Jahren jedoch keinen Einfluss auf die pulmonale Hämodynamik oder die Prognose [14].

Die ärztliche Verordnung der Langzeit-Sauerstoff-Therapie sollte neben den Blutgasbefunden in Ruhe und unter Belastung (mit/ ohne Sparventil) die genauen Angaben der $\mathrm{O}_{2}$-Flussrate in $\mathrm{l} / \mathrm{min}$ sowie die Bezeichnung des Sauerstoffsystems enthalten. In der
Regel wird bei mobilen Patienten ein Flüssigsauerstoffapplikationssystem mit mobilem Zusatzteil oder ein mobiler Konzentrator zum Einsatz kommen. Die Verwendung sog. Spar- oder Demandsysteme mit inspiratorisch getriggerter $\mathrm{O}_{2}$-Freisetzung erlaubt die Einsparung von $\mathrm{O}_{2}$. Die Applikation von Sauerstoff erfolgt in der Regel über Nasensonden. Eine weitere Möglichkeit besteht in der transtrachealen Applikationsweise des Sauerstoffs (Scoop-Katheter), die ebenfalls eine Minderung des $\mathrm{O}_{2}$-Verbrauchs ermöglicht [15]. Bei Patienten, die hohe Flussraten für eine ausreichende Substitution benötigen, kann auch eine größerlumige Sauerstoffbrille, z.B. Oxymizer Pendant ${ }^{\circledR}$, sehr hilfreich sein [16].

Generell ist selbstverständlich die Kooperationsfähigkeit des Patienten Voraussetzung für eine erfolgreiche Durchführung der LTOT. Verlaufskontrollen der Blutgasanalyse zur eventuellen Anpassung der LTOT sowie eine Schulung der Patienten werden als dringend notwendig erachtet [17]. Ob Patienten, die weiter rauchen, von der Therapie ausgeschlossen werden sollten, ist immer wieder Gegenstand der Diskussion. Zweifellos stellt sich hier die Sinnfrage, juristisch existiert eine Grauzone. In jedem Fall ist es ratsam, sich von rauchenden LTOT-Patienten die durchgeführte Information hinsichtlich der Risiken einer Explosionsgefahr bei gleichzeitiger Anwendung von Rauchen und LTOT gegenzeichnen zu lassen.

Entsprechend der Leitlinien ist der Einsatz einer Langzeit-Sauerstoff-Therapie bei einem oder mehreren der folgenden Kriterien indiziert:

- Ruhe- $\mathrm{PaO}_{2}<55 \mathrm{mmHg}$ mit und ohne Hyperkapnie (mindestens 3 mal in 4 Wochen)

- Ruhe- $\mathrm{PaO}_{2}$-Werte zwischen 55 und $59 \mathrm{mmHg}$ bei Nachweis einer pulmonal-arteriellen Hypertonie, peripheren Ödemen als Hinweis auf eine Rechtsherzinsuffizienz oder eine Polyglobulie

- Abfall des $\mathrm{PaO}_{2}$ auf weniger als $55 \mathrm{mmHg}$ bei körperlichen Belastungen, die Aktivitäten des täglichen Lebens entsprechen

- Hypoxämie während des Schlafs

- Verbesserung der körperlichen Belastbarkeit unter LTOT

\section{Welches Gerät für welchen Patienten?}

\section{Immobile Patienten}

Immobile Patienten sind mit einem stationären Sauerstoffkonzentrator grundsätzlich gut versorgt. Die Geräte sind sehr schwer und ausschließlich für den Heimgebrauch geeignet. Der Aktionsradius des Patienten ist vom Konzentrator sowie der jeweiligen Schlauchlänge abhängig.

\section{Alltagsmobile Patienten}

Mobile Patienten, die mehrere Stunden am Tage unterwegs sind, benötigen entweder eine Funktionseinheit aus einem stationären Flüssigsauerstofftank sowie einem mobilen tragbaren System oder einen mobilen Konzentrator [17,18,19]. Die neu auf dem Markt befindlichen Minikonzentratoren weisen den entscheidenden Vorteil auf, dass sie den Patienten unabhängig von der Versorgung mit Flüssigsauerstoff machen und überall betrieben werden können, wo Strom verfügbar ist. Dies schließt bei vielen Systemen auch den Betrieb im PKW ein. Mobile Konzentratoren haben jedoch auch Nachteile: Fast alle mobilen Konzentratoren sind mit einem Demandsystem ausgestattet, nur wenige Systeme gewährleisten wahlweise auch einen Dauerfluss von Sauerstoff [18]. LeBlanc sowie Chatburn u. Mitarb. konnten anhand eines 
Vergleichs mobiler Sauerstoffkonzentratoren zeigen, dass aufgrund unterschiedlicher technischer Spezifikationen (Bolusvolumen, Triggerschwelle etc.) nicht ohne Weiteres von einer vergleichbaren Oxygenierung der Patienten ausgegangen werden kann [20, 21]. Mobile Konzentratoren sind in der Regel nicht geeignet für Patienten, die einen Flow von $>41 /$ min benötigen. Ein Nachteil der Minikonzentratoren ist zudem deren Lautstärke sowie die geringe Akkuleistung.

\section{Spar- oder Demandsysteme - ein wenig beachtetes Problem! \\ $\nabla$}

Sparsysteme verfügen über ein Ventil, das sich nur bei der Inspiration öffnet und entsprechend den Vorgaben - Inspirationszeit, Triggerschwelle, Bolusgröße - eine bestimmte Menge an Sauerstoff abgibt. Bei der Ausatmung verschließt sich das Ventil. Sparventile sind prinzipiell sinnvoll, können aber bei sauerstoffbedürftigen Patienten auch zu einer Gefährdung führen. Die „Demandfähigkeit" muss vor dem dauerhaften Einsatz getestet werden, denn nicht jeder Patient ist in der Lage, die Öffnung des Ventils, insbesondere bei körperlicher Belastung, adäquat zu triggern, da Patienten dann oft zur Mundatmung neigen, sodass der inspiratorische Trigger nicht ausgelöst wird und somit überhaupt keine Sauerstoffabgabe stattfindet [18]. Patienten mit fortgeschrittener Lungenerkrankung sind zweifellos Risikopatienten, bei denen es zu drastischen Sauerstoffunterversorgungen mit Auftreten von zerebralen und kardialen Ischämien sowie Herzrhythmusstörungen kommen kann. Insbesondere unter körperlicher Belastung, bei Zunahme des peripheren $\mathrm{O}_{2}$-Bedarfs und erhöhter Atemfrequenz, kann die Hypoxämie oft nicht mehr kompensiert werden. Dieser wichtige Aspekt wird häufig nicht bedacht! Die klinische Praxis zeigt, dass dies insbesondere für Patienten mit Lungengerüsterkrankungen gilt. Solche Patienten neigen bei Belastung zu erheblicher Tachypnoe.

Die Vielzahl der Demandsysteme stellt aufgrund der unterschiedlichen Parametervorgaben ein Problem bzw. eine oft schwer lösbare Herausforderung für den Patienten, den Arzt und den Versorger dar. Sauerstoff ist ein Medikament, das bei jedem Patienten individuell - und vom Arzt verordnet! - für Ruheund Belastungsphasen dosiert werden muss. Dabei ist nicht zu tolerieren, dass Systeme seitens der Provider oder der Krankenkassen einfach ausgetauscht oder gewechselt werden. Wenn ein Patient auf ein mobiles Flüssigsauerstoffsystem mit Demandsystem titriert wurde, so bedeutet das eben nicht, dass dieses System - ohne erneute $\mathrm{O}_{2}$-Testung! - durch einen mobilen Konzentrator ersetzt werden kann. Es ist auch nicht damit getan, den Ruhebedarf eines Patienten an Sauerstoff zu ermitteln und den Bedarf unter Belastung stereotyp mit einem Pauschalwert „Ruhewert \pm 2 l“ festzulegen.

Patienten mit LTOT sollten bezüglich der Therapie ausreichend informiert und geschult werden. Die Ergebnisse der Compliancestudien belegen, dass ein erhebliches Informationsdefizit bei den Patienten vorliegt $[22,23]$. Bei Kontrolluntersuchungen gilt es zu klären, ob eine adäquate $\mathrm{O}_{2}$-Versorgung des Patienten (auch mit einem Sparventil) gewährleistet bleibt. Der Einsatz von Demandsystemen ist sowohl bei der Flüssigsauerstoff- als auch bei der Konzentratortherapie kritisch zu begleiten und zu kontrollieren. Die Demandfähigkeit sollte vom Arzt im Sauerstoffpass - soweit vorhanden! - fixiert werden.

\section{Compliance \\ $\nabla$}

Den meisten Patienten mit einer Langzeit-Sauerstoff-Therapie ist nicht bewusst, dass es sich bei der Applikation von Sauerstoff um eine medikamentöse Therapie handelt. Diese Therapie kann jedoch, ebenso wie die antiobstruktive bei COPD, nur dann effektiv sein, wenn eine regelhafte und korrekte Applikation erfolgt. Viele Patienten sind der Auffassung, dass Sauerstoff abhängig macht, demzufolge erfolgt auch keine adäquate Nutzungsdauer. Katsenos und Constantopoulos haben in ihrer Übersichtsarbeit die Faktoren identifiziert, die zu einer vergleichsweise schlechten Therapiecompliance führen [21]: Neben dem Abhängigkeitsgefühl sind dies die Nebenwirkungen (trockene Schleimhaut, Nasenbluten etc.), eine falsche Erwartungshaltung hinsichtlich des Therapiebenefits sowie ein Schamgefühl gegenüber der Öffentlichkeit (Stigmatisierung). Der Arzt sollte den Patienten hinsichtlich falscher Erwartungen „Ich nehme Sauerstoff, bemerke aber überhaupt keine Verbesserung meines Befindens“ aufklären. Compliance-mindernd ist, dass vor allem COPD-Patienten oft keine unmittelbare Verbesserung des Befindens bzw. der Leistungsfähigkeit erleben.

Den Literaturangaben zufolge ist die Therapiecompliance hinsichtlich der LTOT daher eher schlecht $[21,22]$. Berücksichtigt man die enormen Kosten der Sauerstofftherapie, so ist hier Handlungsbedarf gegeben. Es erscheint einleuchtend, dass die tägliche Alltagsmobilität eines Patienten mit chronisch respiratorischer Insuffizienz abhängig ist von der Ausprägung des pulmonalen Krankheitsbildes. Bei der Auswahl eines Therapiegerätes sollte zunächst der noch für möglich gehaltene individuelle Mobilitätsgrad ermittelt werden. Je stärker sich die Therapie an den Alltagsbedürfnissen des Patienten ausrichtet, desto besser wird die Compliance sein. Ein bislang unterschätztes Problem stellt die verwirrende Vielfalt der Applikationssysteme und Sparventile dar. Die individuelle Patienteneinstellung auf Sauerstoff sowie eine begleitende Geräteeinweisung sind notwendige Voraussetzungen für eine erfolgreiche Langzeit-Sauerstoff-Therapie. Die Aufgabe des Arztes ist es, den Patienten zu begleiten sowie die medikamentöse Therapie und die LTOT zu überwachen.

Die klinische Praxis zeigt nach wie vor ein erhebliches Maß von sogenannten under-/over- bzw. non-usern. Unter gesundheitsökonomischen Gesichtspunkten wäre es daher empfehlenswert, einer aufwendigen differenzialdiagnostischen LTOT-Indikationsabklärung und deren stetigen Überprüfung im weiteren Verlauf durch angemessene Entgeltpauschalen Rechnung zu tragen. Wenn dadurch die Zahl der korrekten LTOT-Benutzung steigt, Nichtnutzer identifiziert werden und durch Schulung und Motivation von der Notwendigkeit einer LTOT überzeugt werden können, dann wird sich der nicht unbedeutende wirtschaftliche Ressourceneinsatz lohnen. Die Beendigung von im Alltag nicht genutzten LTOT-Verordnungen würde den wirtschaftlichen Spielraum für solche Entgelte schaffen können.

\section{Durchführung einer Sauerstofftitration $\nabla$}

Es ist verständlich, dass der Bedarf an Sauerstoff unter körperlicher Belastung ein erheblich größerer sein wird als der in Ruhe oder im Schlaf. Die Messungen zur bestmöglichen Einstellung eines Patienten mit chronisch respiratorischer Insuffizienz sollten deshalb in Ruhe und unter Belastung vorgenommen werden. $\mathrm{Zu}$ prüfen ist insbesondere, ob der mobile Patient, der auf ein Demandsystem eingestellt werden soll, unter Belastung ausrei- 


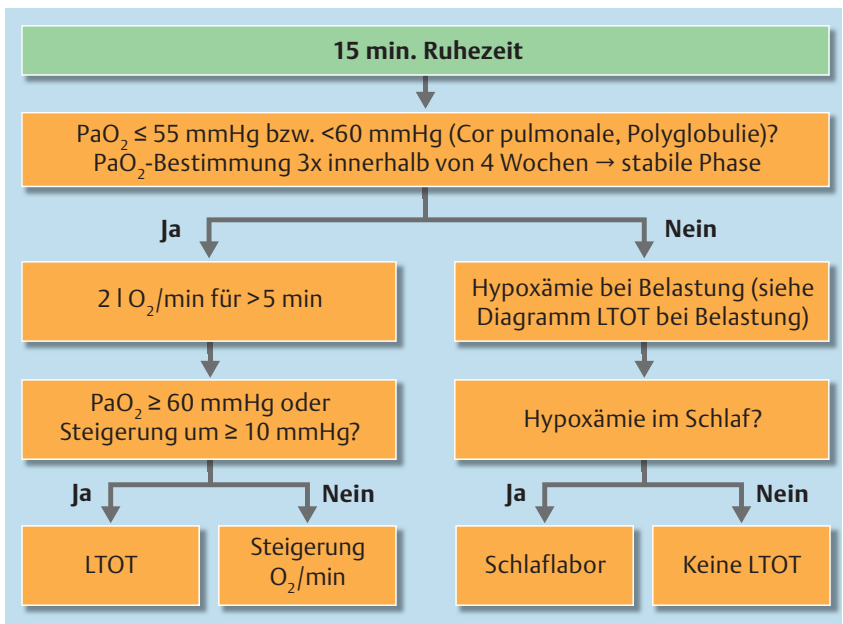

Abb. 1 Langzeit-Sauerstoff-Therapie (LTOT).

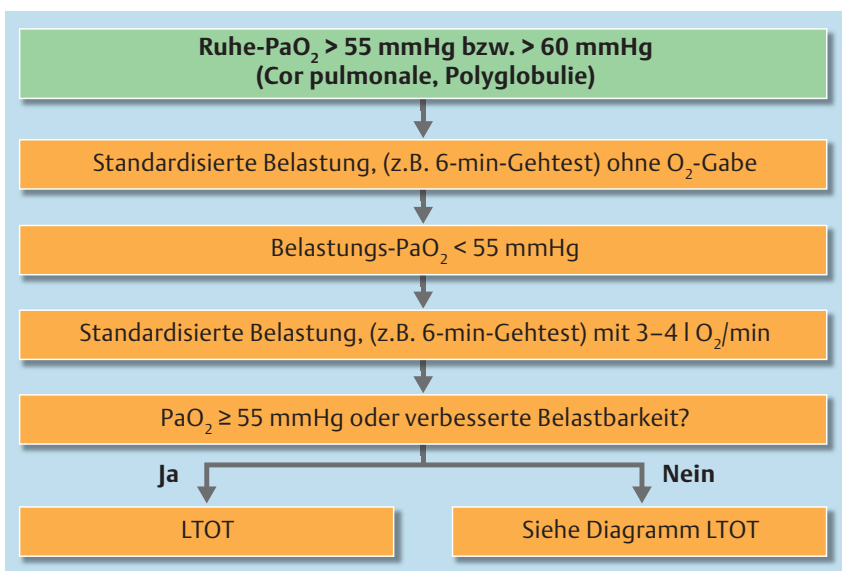

Abb.2 Langzeit-Sauerstoff-Therapie (LTOT) bei Belastung.

chend oxygeniert wird. Die belastungsinduzierte Hypoxämie sollte z. B. mittels eines 6-Minuten-Gehtests objektiviert werden. Ziel muss es sein, das richtige $\mathrm{O}_{2}$-System mit einer ausreichenden Zielsättigung während der gesamten $\mathrm{O}_{2}$-Therapiephase für den Patienten vorzuhalten. Auch die Schlafphase sollte in die dauerhafte Sauerstoffversorgung miteinbezogen werden, stellt sie doch eine Zeitspanne von etwa 6 bis 8 Stunden mit veränderter Atemtiefe dar. Wie sollte nun eine optimale Einstellung des individuellen Sauerstoffbedarfs durchgeführt werden?

\section{Blutgasanalyse in Ruhe ( 0 Abb. 1)}

Nach einer Ruhephase von 15 min Durchführung einer BGA unter Raumluftatmung mittels Blutentnahme aus dem hyperämisierten Ohrläppchen. Wenn der $\mathrm{PaO}_{2} \leq 55 \mathrm{mmHg}$, Applikation von $21 \mathrm{O}_{2} /$ min über eine Nasensonde über eine Zeitdauer von $>5$ min. Dann erneute Durchführung einer BGA. Die Sauerstoffgabe sollte $\mathrm{zu}$ einem Anstieg des $\mathrm{PaO}_{2} \geq 60 \mathrm{mmHg}\left(\mathrm{SaO}_{2} \geq 90 \%\right)$ führen. Bei nicht ausreichendem Anstieg Steigerung der $\mathrm{O}_{2}$ Flussrate auf 3 oder 41/min.

\section{Blutgasanalyse unter Belastung ( $($ Abb.2)}

Durchführung einer Belastung (z.B. 6-Minuten-Gehtest oder Ergometertest) ohne $\mathrm{O}_{2}$-Gabe. Der Patient wird aufgefordert, eine markierte Wegstrecke in einem für sich normalen Tempo 6 Minuten lang zu gehen. Die zurückgelegte Wegstrecke und die
Bestimmung der Blutgase nach der Belastung geben dem Arzt eine Information darüber, ob überhaupt die Notwendigkeit der Einleitung einer mobilen LTOT besteht. Zur Beurteilung subjektiv empfundener Atemnot sollte der Patient diese auf einer BorgSkala bewerten. Wenn der $\mathrm{PaO}_{2}$ unter Belastung auf $\leq 55 \mathrm{mmHg}$ fällt, empfiehlt sich die Applikation von 3-41 $\mathrm{O}_{2} /$ min über eine Nasensonde und eine erneute Bestimmung der Gehstrecke, der Blutgaswerte und der Dyspnoe am Ende der Belastung. Bei integriertem Demandsystem ist selbstverständlich ebenfalls eine Überprüfung der Gehstrecke und Analyse der BGA vor und unter Belastung notwendig. Während der Belastungsphase besteht die Option der kontinuierlichen Dokumentation der Sauerstoffsättigung via Pulsoxymeter.

\section{Blutgasanalyse im Schlaf}

Eine Bestimmung der Sauerstoffsättigung (Pulsoxymeter) in der Nacht sollte ohne und mit Gabe von 21 Sauerstoff erfolgen.

Fazit

$\nabla$

1. Sauerstoffgabe ist eine medikamentöse Therapie, die bei nicht adäquater Applikation den Patienten akut und chronisch ge-

fährden kann.

2. Die LTOT (stationär/mobil und Flüssigsauerstoff/Konzentrator) sollte dem Patienten individuell angepasst werden.

3. Vor Einleitung einer mobilen LTOT bedarf es der arteriellen bzw. kapillär durchgeführten Blutgasanalyse in Ruhe und nach Belastung.

4. Der Einsatz von Spar- bzw. Demandventilen ist kritisch zu prüfen. Er ist nur aufgrund ärztlicher Verordnung gerechtfertigt und bedarf engmaschiger Kontrollen!

5. Die LTOT sollte primär durch einen Lungenfacharzt verordnet werden.

6. Eine Umstellung mobiler Flüssigsauerstoffsysteme auf miniaturisierte Konzentratoren darf - aufgrund der unterschiedlichen Systemkonfigurationen - nur nach Durchführung von erneuten Blutgasanalysen durch den Arzt vorgenommen werden.

\section{Abkürzungen \\ $\nabla$ \\ LTOT Long Term Oxygen Therapy (Langzeit-Sauerstoff-Therapie) \\ COPD chronic obstructive pulmonary disease}

\section{Interessenkonflikt}

$\nabla$

Die Autoren geben an, dass kein Interessenkonflikt besteht.

\footnotetext{
Institute

${ }^{1}$ Klinik für Innere Medizin, SP Pneumologie, Intensiv- und Schlafmedizin (Direktor: Prof. Dr. C. Vogelmeier), Philipps-Universität Marburg

2 Medizinische Informatik, Fachbereich MNI (Mathematik, Naturwissenschaft, Informatik), Technische Hochschule Mittelhessen, Gießen

${ }^{3}$ Medizinische Klinik II, Innere Medizin und Pneumologie, Lungenzentrum Saar, SHG-Kliniken Völklingen

${ }^{4}$ Schön Klinik Berchtesgadener Land, Schönau
} 


\section{Literatur}

1 Koehler U, Greib C, Holland A et al. Therapeutische Optionen bei chronisch respiratorischer Insuffizienz. Internist 2001; 42: 363-372

2 Becker HF, Piper AJ, Flynn WE et al. Breathing during sleep in patients with nocturnal desaturation. Am J Respir Crit Care Med 1999; 159: $112-118$

3 Schäfer H, Ewig S, Hasper E et al. Schlafbezogene Atmungsstörungen bei chronisch obstruktiver Atemwegserkrankung (COPD): Diagnostische und therapeutische Konsequenzen. Pneumologie 1996; 50: $278-285$

4 Kenn K, Heinzelmann I. Rehabilitation in der Pneumologie. Dtsch med Wochenschr 2012; 137: 1575-1578

5 Koehler U, Koehler KI, Vogelmeier C et al. Sport activities of patients with severe COPD in an outpatient setting - only possible with self-guidance? Pneumologie 2010; 64: 194-195

6 Medical Research Council Working Party. Long term domiciliary oxygen therapy in chronic hypoxic cor pulmonale complicating chronic bronchitis and emphysema. Lancet 1981; 1: 681-686

7 Nocturnal Oxygen Therapy Trial Group. Continuous or nocurnal oxygen therapy in hypoxemic chronic obstructive lung disease. Ann Intern Med 1980; 93: 391 - 398

8 Weitzenblum E, Sautegeau A, Ehrhart $M$ et al. Long-term oxygen therapy can reverse the progression of pulmonary hypertension in patients with chronic obstructive pulmonary disease. Am Rev Respir Dis 1985; 131: $493-498$

9 Zielinski J, Tobiasz M, Hawrylkiewicz I et al. Effects of long-term oxygen therapy on pulmonary hemodynamics in COPD patients: a 6-year prospectice study. Chest 1998; 113: 65 -70

10 Dubois P, Jamart J, Machiels J et al. Prognosis of severely hypoxemic patients receiving long-term oxygen therapy. Chest 1994; 105: 469-474

11 Chailleux E, Fauroux B, Binet $F$ et al. Predictors of survival in patients receiving domiciliary oxygen therapy or mechanical ventilation. A 10year analysis of ANTADIR observatory. Chest 1996; 109: 741 - 749
12 Magnussen H, Kirsten AM, Köhler D et al. Leitlinien zur Langzeit-Sauerstofftherapie. Pneumologie 2008; 62: 748 - 756

13 AARC Clinical Practice Guideline. Oxygen Therapy in the Home or Alternate Site Health Care Facility - 2007 Revision and Update. Respir Care 2007; 52: $1063-1068$

14 Chaouat A, Weitzenblum E, Kessler R et al. A randomized trial of nocturnal oxygen therapy in chronic obstructive pulmonary disease patients. Eur Respir J 1999; 14: 1002 - 1008

15 Christopher KL, Schwartz MD. Transtracheal oxygen therapy. Chest 2011; 139: $435-440$

16 Kenn $K$. The effect of an Oxymizer Pendant on oxygenation in patients with interstitial lung diseases or COPD. AJRCCM 2012; abstract

17 Dunne PJ. The Clinical Impact of New Long-Term Oxgen Therapy Technology. Respir Care 2009; 54: 1100-1111

18 Dunne PJ, MacIntyre NR, Schmidt UH et al. Respiratory Care Year in Review 2011: Long-Term Oxygen Therapy, Pulmonary Rehabilitation, Airway Management, Acute Lung Injury, Education, and Management. Respir Care 2012; 57: 590-606

19 Dunne PJ. Long-term oxygen therapy (LTOT) revisited: In defense of non-delivery - technology. Rev Port Pneumol 2012; 18: 155 - 157

20 LeBlanc CJ, Lavallee LG, King JA et al. A comparative study of 3 portable oxygen concentrators during a 6 -minute walk test in patients with chronic lung disease. Respiratory Care 2013; 58: 1598-1605

21 Chatburn RL, Williams TJ. Performance comparison of 4 portable oxygen concentrators. Respiratory Care 2010; 55: 433-442

22 Katsenos S, Constantopoulos SH. Long-Term Oxygen Therapy in COPD: Factors Affecting and Ways of Improving Patient Compliance. Pulmonary Medicine 2011; 2011: 325362

23 Arnold E, Bruton A, Donovan-Hall $M$ et al. Ambulatory oxygen: why do COPD patients not use their portable systems as prescribed? A qualitative study. Pulmonary Medicine 2011; $11: 9$ 